\title{
An Enhancement of Roentgenogram by Using Image Processing Techniques
}

\author{
Yogesh Singh ${ }^{1}$, Sangeeta Aneja ${ }^{2}$, Santosh Singh $^{3}$, Keerti Mathur ${ }^{4}$ \\ ${ }^{1,4}$ Department of Physiology, SMS Medical College, Jaipur \\ ${ }^{2}$ Department of Physiology \& Radiodiagnosis, SMMH Medical College, Saharanpur \\ ${ }^{3}$ Department of Computer Science, Banasthali University, Banasthali \\ Email: ${ }^{1}$ yogeshsingh4u@yahoo.com
}

\begin{abstract}
Chest imaging is a very useful method for looking inside the organ for assessing the scenario with noninvasive manner. Advance imaging play a significant role by introducing newer techniques day by day, but chest roentgenogram still mark a role in early decision in disease management. Good quality of Chest roentgenogram is available in rural setups without financial overburden to patients. The present study demonstrated that the chest roentgenogram visualization is improving using MATLAB and is giving superior envisage in comparing the conventional one. Better quality of Chest X-ray is more helpful to physicians for early diagnosis of disease. The early detection of disease is becoming more efficient and improving the quality of life.
\end{abstract}

Keywords: Roentgenogram, Visualization, Early diagnosis, Disease

\section{INTRODUCTION}

In the developing countries more expensive advance testing is considered a financial burden to the populace as well as on the budget of the Government. Conventional X-ray imaging is the most popular and less expensive diagnostic test since it was discovered.

Since 1895 day to day improvement has brought in this test as it is a first line investigation, when the person goes to any medical center with chest discomfort. It is the least expensive and quick diagnostic imaging system which is available in rural medical setup so the chest X-ray imaging (Roentgenogram) is the most common test [2]. Just as the coin has two faces similarly the advance facilities have some advantages and disadvantages.

Digitalization of the chest X-ray imaging is the advance step of the conventional X-ray imaging. Production of digital X-rays from pictorial radiographs is becoming a common practice to maximize information and reduce the number of rejected X-rays. Advance digitization trim down the film processing time and can be easily handled but some unwanted information is added during acquisition process and thus digitization is the main source of noise in digital X -ray images [6].

This surplus information deviate the observer from the right pathway of disease management. Image processing helps to suppress the surplus information and highlights the meaningful information for achieving the goal. Image processing is a tool for highlighting the pixel information in a better way so the reader can recognize the meaningful information and make a decision for further treatment.

The conventional chest X-ray film is totally dependent on hand of expertise. If the technician is well experienced, the output of the film is better and the expertise physician can read meaningful information within less time. On the other hand if the quality of film is poor, the expertise readers face a difficulty to read out film with lots of unwanted information, which may sometimes lead to misdiagnosis of disease. To avoid such type of cases, image needs to be enhanced. Image processing helps to enhance the image which gives meaningful information after applying histogram equalization and filtering process. The present study gives an impetus to highlight the role of enhanced imaging for early detection of disease and disease management in a less time. This golden period increases the rate of survival. 


\section{REVIEW OF LITERATURE}

Li et al proposed an improved segmentation method on set of chest radiographs based on statistical shape and appearance models. For the shape model, multi-scale and multi-step-size with different limitation parameters were used to increase the searching ability. For the appearance model, multiple features with different weights were used to describe different parts of the lung field border [8].

Pre and post processing of digital image analysis helps to reduce perceptual and cognitive errors by highlighting abnormalities and characterizing lung patterns. It works as effective second opinion to support physicians. Preprocessing of the digital images is necessary as they are prone to a variety of noises [4], [10].

Kale Vaishanw G studied on radiological lungs X-ray image and highlighted the effect of spatial filtering. This results in taking decision for applying the proper spatial filtering method to enhance the image and also highlight the effect of spatial filtering on radiological X-ray image of lungs. [7].

To get the better quality and contrast enhancement of the image, Raihan Firoz et al carried out morphological transform operation by using disk shaped mask [11].

Udeshani et al demonstrated a novel approach to use a pipeline of image processing routines to remove noise and segment the lung from other anatomical structures in the chest X-ray and extract regions that exhibit shape characteristics of lung nodules. The proposed approach detected nodules in the diseased area of the lung with an accuracy of $96 \%$ using the pixel-based technique while the feature-based technique produced an accuracy of $88 \%$ [12].

\section{METHODOLOGY}

The Digital Imaging and Communications in Medicine (DICOM) images are stored in the storage drive for analysis and further processing. During the storing process of X-ray image, a lot of irrelevant information is added. This irrelevant information also contains noise. Secondary radiation, development and handling of film of X-ray and alteration of graphic X-ray to digital are the key basis of noise [6]. All this affects the quality of the image and reduces the contrast in the image.

The dilemma with these images is that at a time, they may be noisy, blurred, and improperly bright and out of focus, which makes the visualization more difficult. The level of pixel intensity is another problem, which is same for the tissues. One of the solutions to all above problems can be image enhancement. Although it does not give us the assurance of high quality outcomes but it reduces the noise and also improves the contrast [7]. These images are imported into the Computer Aided Detection (CAD) system [13].Before applying any segmentation and feature extraction algorithm, image enhancement is done to produce more effective image both in terms of cost and quality. It also reduces the repeated exposure to radiation on the subject

The main aim of enhancement of the image is to emphasize the features of the image for successive analysis. It simply emphasizes certain specified image characteristics.

Image enhancement improves the contrast as well as enriches the edges. Enhancement also includes sharpening, filtering, pseudo coloring etc. This process is very useful in analyzing the image as well as in feature extraction. It shows the improved information of the image that emphasizes certain precise characteristic features of the image [9].

This process starts with the conversion of the original image to gray scale image. Grayscale image is also known as black and white image and each pixel has only one value which is the intensity of the pixel. The value may range from 0 to 255, varying from white at the strongest intensity to black at the weakest [3]. Fig 1 shows the original image, Fig 2 shows the gray scale image and fig 3 shows its corresponding histogram i.e. pictorial representation of the distribution of pixel intensity values.

To obtain the uniform histogram, histogram equalization is applied [Fig 4, 5]. In this technique image intensities are adjusted to enhance contrast which helps the radiologists as well as physicians to visualize more information. It provides improved vision in X-ray films. It is an important but a challenging task to maintain the details of the image while smoothing the noise using filtering. It can only be done by median filter i.e. non linear filter. The edges 
of the image are preserved even after the changes in the values of pixel intensities by using median filter. It is effective in removing impulses and also smoothens additive white noise [4], [5].

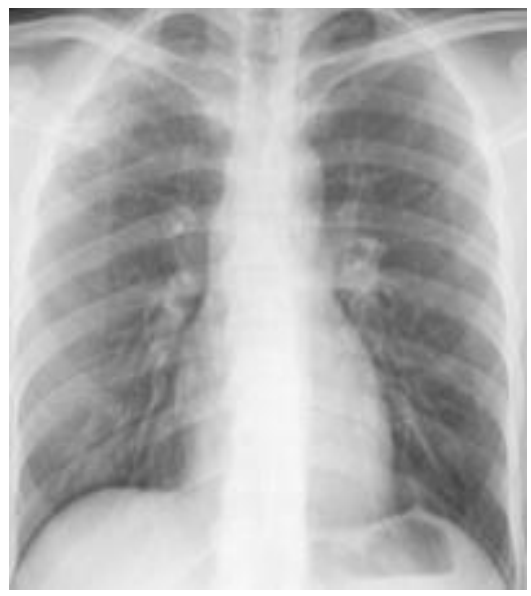

Fig. 1 Original Image

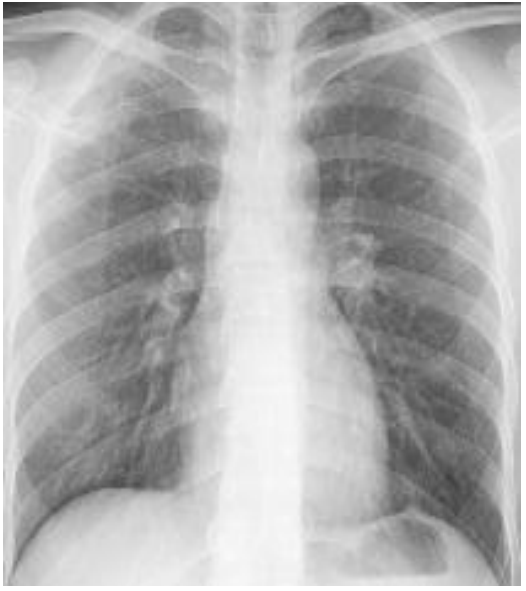

Fig. 2 Gray Scale Image

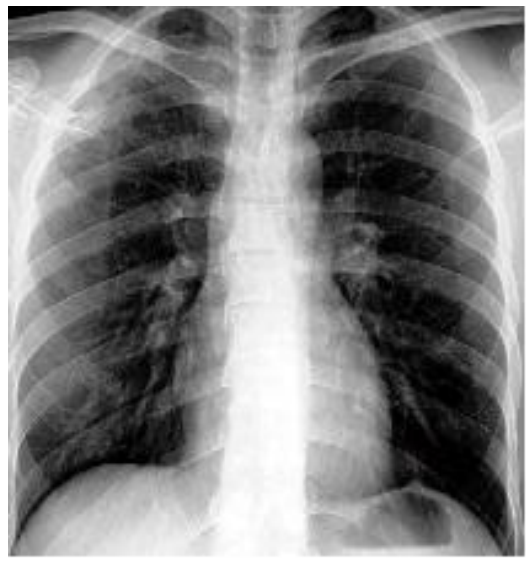

Fig. 4 Histogram Equalized Image

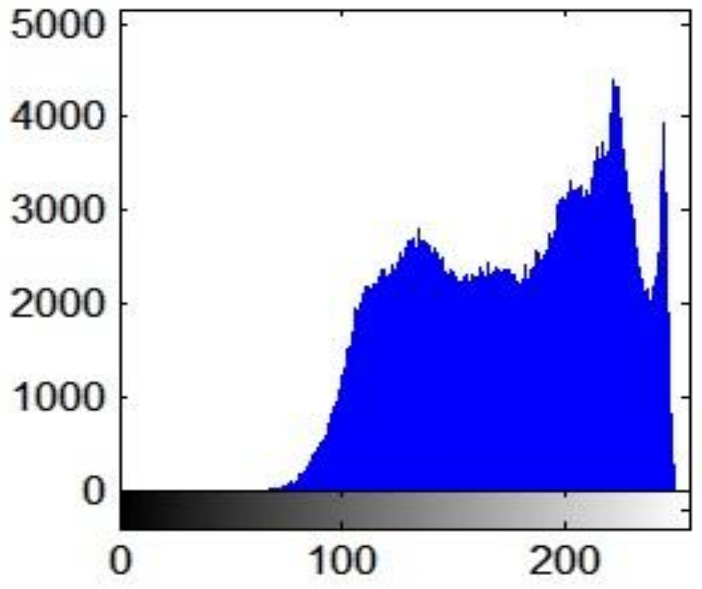

Fig. 3 Histogram of fig. 2

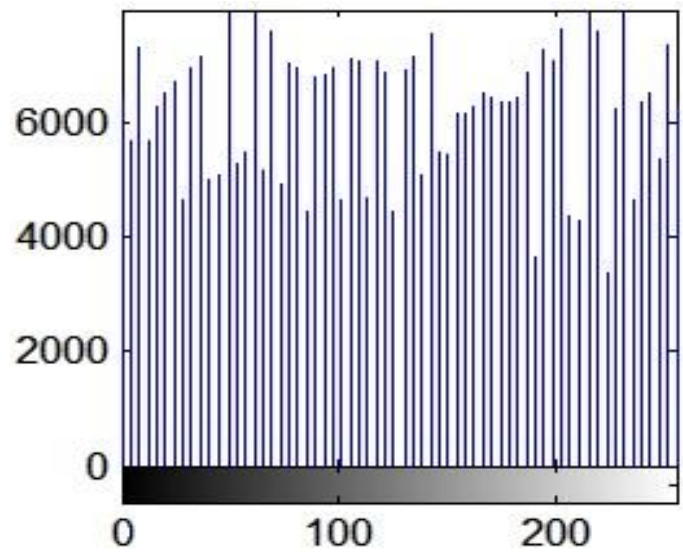

Fig. 5 Histogram of fig. 4 


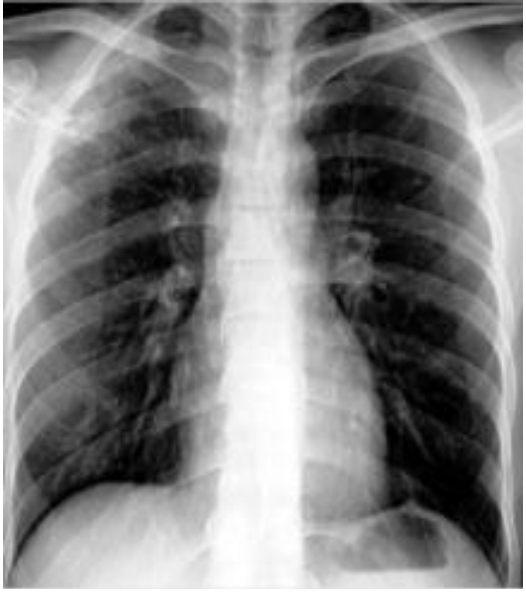

Fig. 6 Enhanced Image

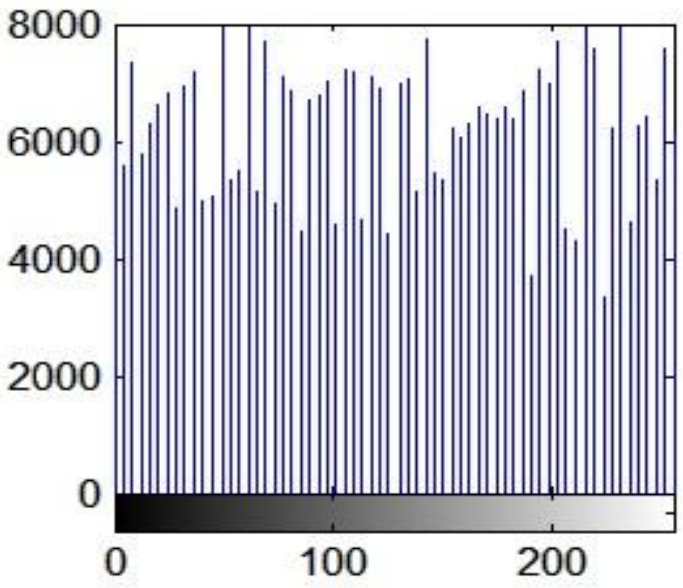

Fig. 7 Histogram of fig. 6

In median filter, the value of the output pixel is determined by the median of the neighboring pixel values rather than mean. It replaces the center value in the kernel with calculating median of all pixels around the kernel. This filter is therefore better able to remove this outlier as it has advantage of keeping the sharpness of the image. An example of median filtering of single $3 * 3$ values is shown below

Table I Unfiltered values

\begin{tabular}{|l|l|l|}
\hline 50 & 70 & 65 \\
\hline 70 & 30 & 35 \\
\hline 60 & 70 & 40 \\
\hline
\end{tabular}

Median $=30,35,40,50,60,65,70,70,70=60$

Table II Filtered values

\begin{tabular}{|c|c|c|}
\hline- & - & - \\
\hline- & 60 & - \\
\hline- & - & - \\
\hline
\end{tabular}

Center value is replaced by median of all values. Median filter processing technique is employed to remove this irrelevant information and for enhancing the X-ray image. Advantage of using this filter is the preservation of edges [1].

This study introduces the painless enhancement techniques using MATLAB tool with significant output and demonstrates the superior image quality, structural manifestation of input image. Noises were also removed from an input image.

\section{CONCLUSION}

Image processing and analysis provide a means to extract and quantify objects and patterns to obtain the relevant information from the image. That meaningful information is helpful to radiologists as well as 
physicians to make a map for treatment without further delay. But during the capturing of the image some redundant information is added which is the cause of misdiagnosis or failure to spot the golden period. That golden period is very important for increasing the rate of survival as well as to reduce the repeated exposure due to poor quality of X-ray film.

\section{REFERENCE}

[1] Amal M. Al Gindi, Tawfik A. Attiatalla and Moustafa M. Sami. Journal of American Science 2014; 10(6):13-22.

[2] Fatemeh Shahsavari Alavijeh and Homayoun Mahdavi-Nasab. "Multi-scale Morphological Image Enhancement of Chest Radiographs by a Hybrid Scheme", J Med Signals Sens. 2015 Jan-Mar; 5(1): 59-68.

[3] Gonzalez RC, Woods RE. Ch. 4. MA: Addison-Wesley Publishing Company; 1992. Digital Image Processing.

[4] Gupta G, Algorithm for image processing using improved median filter and comparison of mean, Median and improved median filter, International Journal of soft Computing and Engineering, Vol 1 (5), 2011, 304-311.

[5] Hall EH. Almost uniform distribution for computer image enhancement. IEEE Trans Comput. 1974; $23: 207-8$.

[6] Jassam, Khalil, I. 1992, Image Processing of Conventional X-ray, International society of Photogrammetry and Remote Sensing.

[7] Kale Vaishanw G, X-Ray Lung Image Enhancement by Spatial Filtering. International Journal of Innovative Research in Science, Engineering and Technology, Vol 3 (4), 2014, 330-336.

[8] Li, Xuechen; Luo, Suhuai; Hu, Qingmao; Li, Jiaming; Wang, Dadong; Chiong, Fabian, Journal of Medical Imaging and Health Informatics, Volume 6, Number 2, April 2016, pp. 338-348.

[9] Nirmala D, Medical image contrast enhancement techniques, Journal of Chemical and Pharmaceutical Research, 2015, 7(7):1-8.

[10] Prakash O. and Khare A, Medical Image Denoising Based on Soft Thresholding Using Biorthogonal Multiscale Wavelet Transform, Int. J. Image Grap. Volume 14, Issue 01\&02, 2014.

[11] Raihan Firoz, Md. Shahjahan Ali, M. Nasir Uddin Khan, Md. Khalid Hossain, Md. Khairul Islam, Md. Shahinuzzaman, Medical Image Enhancement Using Morphological Transformation, Journal of Data Analysis and Information Processing, 2016, 4, 1-12.

[12] Udeshani K.A.G., Meegama R.G.N., Fernando T.G.I., Statistical Feature-based Neural Network Approach for the Detection of Lung Cancer in Chest X-Ray Images, International Journal of Image Processing (IJIP), Volume (5): Issue (4) : 2011, 425-434.

[13] Vijay A. Kotkar, Sanjay S. Gharde, Review of Various Image Contrast Enhancement Techniques. International Journal of Innovative Research in Science, Engineering and Technology. Vol. 2, Issue 7, 2013, 2786-93. 